\title{
Tetravalenter Meningokokken-Impfstoff
}

Mit der Zulassung von Nimenrix ${ }^{\mathrm{TM}}$ im April 2012 durch die Europäische Arzneimittelagentur EMA steht ein neuer Meningokokken-Konjugatimpfstoff zur Verfügung, der Kleinkinder, Kinder und Erwachsene vor den 4 Neisseria meningitidis-Serogruppen A, C, W-135 und Y schützen kann [1, 2]. Es ist der erste 4-valente Meningokokken-Konjugatimpfstoff, mit dem bereits Kinder ab dem vollendeten zwölften Lebensmonat geimpft werden können. Er wird als einmalige Impfdosis verabreicht und kann auch Personen appliziert werden, die zuvor schon einmal einen Meningokokken-Polysaccharidimpfstoff erhalten haben [1].

„Grundsätzlich kann jeder an einer Meningokokken-Meningitis erkranken“, erklärte Prof. Dr. Markus Knuf, Direktor der Klinik für Kinder und Jugendliche, Dr. Horst Schmidt Kliniken, Wiesbaden. „In erster Linie trifft die Infektion aber Säuglinge, Kleinkinder und junge Erwachsene. Umso mehr begrüßen wir die Möglichkeit eines erweiterten Schutzes durch den neuen quadrivalenten Impfstoff.“

\section{Schutz vor 4 Stämmen}

Mittlerweile konnten 13 Serogruppen von Neisseria meningitidis identifiziert werden, 6 von ihnen (die Serogruppen A, B, C, W-135, X und Y) verursachen die Mehrzahl der weltweiten Meningokokkeninfektionen [3]. Der neu zugelassene Impfstoff ist zum Schutz vor 4 der 6 krankheitsrelevanten Meningokokkenstämme, die weltweit für die Mehrzahl invasiver Meningokokkenerkrankungen (IME) verantwortlich gemacht werden $[1,2]$. „Meningokokken-Meningitis und Meningokokken-Sepsis sind lebensbedrohliche Erkrankungen, die selbst unter bestmöglicher Behandlung fulminant verlaufen können und oftmals binnen 24 Stunden zum Tode führen“, warnte Knuf. Der Prävention im Sinne einer Impfung komme deshalb hohe Bedeutung zu. Immunogenität und Sicherheit von $\mathrm{Ni}$ menrix ${ }^{\mathrm{TM}}$ wurden in einem umfangreichen klinischen Studienprogramm (17 Studien in 17 Ländern) mit mehr als 8000 Teilnehmern im Alter von 1 bis 55 Jahren untersucht. Der neue Impfstoff wurde hierbei

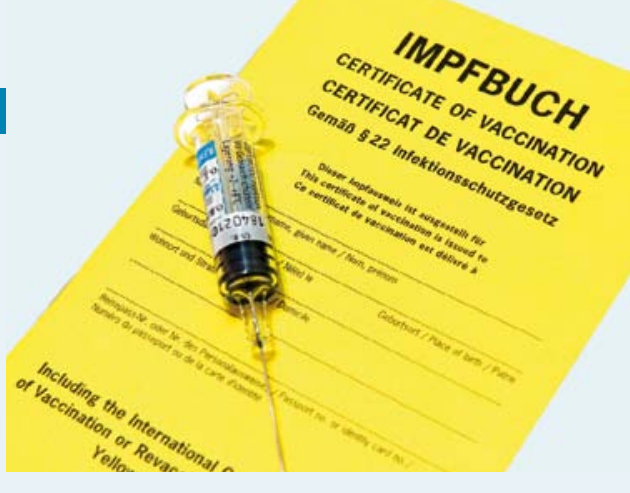

im Allgemeinen gut vertragen. Häufigste Nebenwirkungen waren Appetitlosigkeit, Reizbarkeit, Schläfrigkeit, Kopfschmerzen, gastrointestinale Beschwerden, Fieber, Müdigkeit sowie Lokalreaktionen an der Injektionsstelle (Schmerzen, Rötung, Schwellung, Hämatom) [1].

Die Markteinführung des Impfstoffs ist für Juli 2012 geplant.

\section{Literatur}

$1 \quad$ Nimenrix ${ }^{\mathrm{TM}}$ Fachinformation, April 2012

2 World Health Organization. Meningococcal vaccines: WHO position paper, November 2011. Weekly Epidemiological Record 2011; 86: 521

3 Halperin SA, Bettinger JA, Greenwood B et al. The changing and dynamic epidemiology of meningococcal disease. Vaccine 2011 Dec 15 [Epub ahead of print]

Quelle: Pressemitteilung GlaxoSmithKline, München, 04.05.2012

\section{Information zu Meningokokken-Meningitis}

Anlässlich des Welt-Meningitis-Tages am 24.04. machte Novartis Vaccines auf ein neues Informationsangebot über Meningitis im Internet aufmerksam: Die Webseite, www.meningitis.com/Deutschland/ index.shtml, informiert über MeningitisErkrankungen, Übertragungswege, Risikogruppen und Vorbeugung.
Die deutschsprachige Webseite konzentriert sich vorrangig auf die bakterielle Meningokokken-Infektion, die zu Meningokokken-Meningitis und -Sepsis führen kann. Diese ansteckenden bakteriellen Infektionen werden durch Neisseria meningitidis-Bakterien verursacht. Sie umfassen 12 verschiedene Gruppen (d.h. Serogruppen).

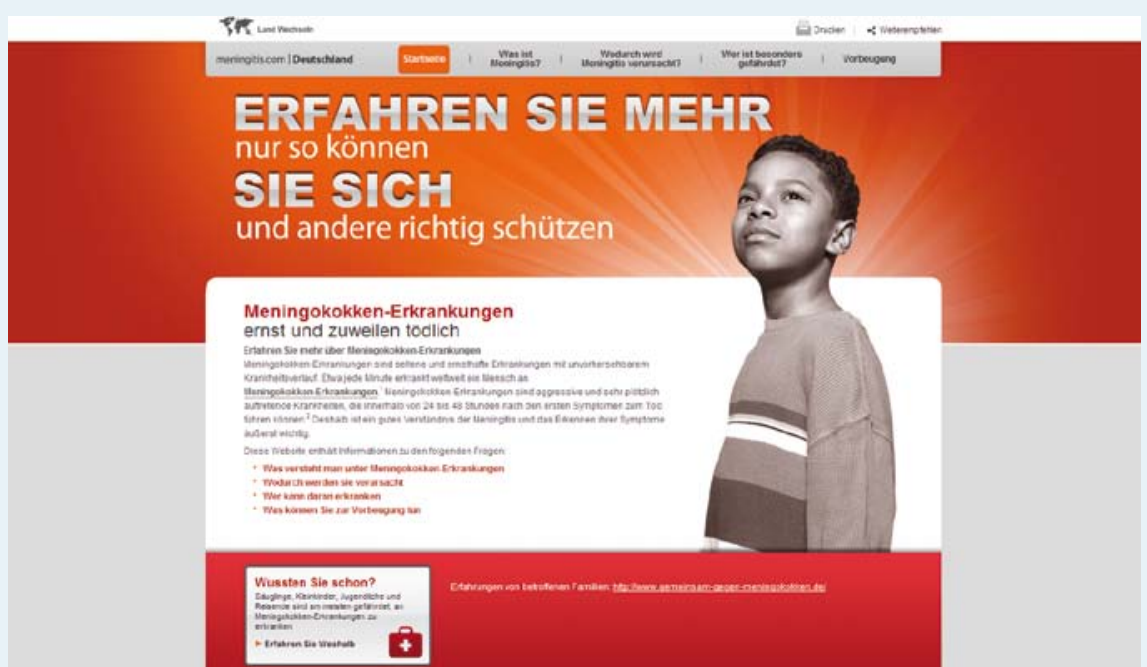

Etwa jede Minute erkrankt weltweit ein Mensch an Meningokokken-Erkrankungen [1]. Die seltenen, aber ernsten Infektionen sind unter Umständen schwer erkennbar. Wenn sie diagnostiziert werden, kommt möglicherweise schon jede Hilfe zu spät. Denn auch wenn die frühen Symptome einer Grippeinfektion ähneln, kann die Erkrankung 24-48 Stunden nach Eintreten der Symptome zum Tod führen [2]. Deshalb sind ein gutes Verständnis der Meningitis und das Erkennen ihrer Symptome äußerst wichtig.

\section{Literatur}

1 Roche P, Spencer J, Marianos A. Editorial: Meningococcal disease. Commun Dis Intell 2001; 25: 126-9

2 Pelton SI. Meningococcal disease awareness: clinical and epidemiological factors affecting prevention and management in adolescents. J Adolesc Health 2010; 46: S9-S15

Quelle: Pressemitteilung Novartis Vaccines Deutschland, Marburg, 23.04.2012 


\section{CRM flexi.LEARN}

Das medizinische Wissen entwickelt sich rasant weiter. Deshalb sind Ärzte verpflichtet, sich regelmäßig fortzubilden und sich über neueste Erkenntnisse zu Krankheiten, Behandlungsmethoden oder Impfungen zu informieren. Doch nicht immer lässt sich der Besuch von Fortbildungsveranstaltungen problemlos mit dem beruflichen und privaten Alltag vereinbaren. Das CRM⿻ Centrum für Reisemedizin bietet Ärzten deshalb jetzt die Mög-疋 lichkeit, sich auf dem Gebiet der Reisemedizin per E-Learning fortzubilden: Der erste Teil des CRM Basisseminars Reise- und\& Tropenmedizin kann in individuell festgelegten Lerneinheiten am eigenen PC absolviert werden. Das Basisseminar entspricht dem Curriculum „Reisemedizinische Gesundheitsberatung“ der है Bundesärztekammer und ist CME-Punkte-zertifiziert.

Flexibles Lernen

CRM flexi.LEARN verbindet die Vorzüge des flexiblen E-Learnings mit den Vorteilen einer Präsenzveranstaltung: Das 32-stündige CRM Basisseminar Reise- und Tropenmedizin besteht aus 2 Lernblöcken. Block eins bietet allgemeine Grundlagen zur Reisemedi- $\frac{\pi}{\Phi}$ zin und zur reisemedizinischen Vorsorgeberatung. Hier werden $\frac{2}{\Phi}$ Kenntnisse zu reiserelevanten Erkrankungen, Impfungen und impfpräventablen Krankheiten, zur Malaria und Vektorprophyla- $\frac{\bar{\Phi}}{8}$ xe sowie zur Nahrungsmittel- und Trinkwasserhygiene vermit- $\frac{\pi}{\Phi}$ telt. Dieser erste Lernblock kann ab sofort als E-Learning-Fortbildung absolviert werden.

Der zweite Block des Basisseminars schließt sich als Präsenzveranstaltung an. Auf dem Lehrplan stehen dann Themen wieల nicht infektiöse Gesundheitsrisiken auf Reisen oder die Grundlagen der Geomedizin. Die Präsenzveranstaltung endet mit ei-৫ ner Prüfung. Bei erfolgreichem Abschluss erhalten die Teilneh- $\frac{-}{\Phi}$ mer das CRM Zertifikat Reise- und Tropenmedizin.

„Mit CRM flexi.LEARN können Mediziner ihre Fortbildung nun:ठ্ sehr flexibel gestalten“, sagt Lothar Münnix, Geschäftsführer $\stackrel{\frac{2}{\varrho}}{\bar{\alpha}}$ des CRM Centrum für Reisemedizin in Düsseldorf. „Die Möglichkeit des E-Learning macht sie örtlich und zeitlich unabhän- $N$ gig, es entfällt eine aufwendige Anreise zum Veranstaltungsort und die damit verbundenen Kosten.“

\section{Umfangreiche Informationen in Online-Bibliothek}

Ähnlich einer konventionellen Fortbildung strukturiert das inter-ฉ̊ netbasierte Computerseminar des CRM die Lerninhalte in verschie- $₫$

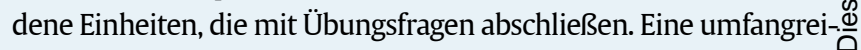
che Online-Bibliothek bietet Hintergrundinformationen $\mathrm{zu}$ Krankheiten und Therapien. In einem integrierten Forum erhalten Teilnehmer Unterstützung bei Fragen zu Inhalten oder zur Technik. Das CRM Basisseminar Reise- und Tropenmedizin, bestehend aus E-Learning-Modul und Präsenzveranstaltung, kann für den Preis von 565 Euro über die Website des CRM unter http://elearning.crm.de gebucht werden. Interessierte Ärzte haben dort die Möglichkeit das E-Learning-Angebot kostenlos und unverbindlich anhand von 2 verschiedenen Themenmodulen zu testen. Interessierte finden außerdem weitere Informationen zum CRM flexi.LEARN, zu den erforderlichen technischen Voraussetzungen und zum Probezugang.

Quelle: Pressemitteilung des CRM Centrum für Reisemedizin, Düsseldorf 